\title{
Quantum Decoherence of Charge Qubit coupled to Nonlinear Nanomechanical Resonator
}

\author{
CHEN Cheng ${ }^{1}$ and GAO Yi-bo', 因 \\ ${ }^{1}$ College of Applied Sciences, Beijing University of Technology, Beijing, 100124, China
}

\begin{abstract}
When the nonlinearity of nanomechanical resonator is not negligible, the quantum decoherence of charge qubit is studied analytically. Using nonlinear Jaynes-Cummings model, one explores the possibility of being quantum data bus for nonlinear nanomechanical resonator, the nonlinearity destroys the dynamical quantum information-storage and maintains the revival of quantum coherence of charge qubit. With the calculation of decoherence factor, we demonstrate the influence of the nonlinearity of nanomechanical resonator on engineered decoherence of charge qubit.
\end{abstract}

PACS numbers: 03.65.Yz, 85.25.Cp, 62.25.-g

Keywords: quantum decoherence, charge qubit, nonlinear nanomechanical resonator, cavity QED

\section{INTRODUCTION}

Any quantum system, is immersed into the environment, can not be isolated from the environment completely. [1] In literatures, one mimics the environment with the "bath" model. The fluctuation of the bath induces the dissipation and decoherence of the quantum system such that long decoherence time is very important for qubit. Actually, to implement quantum computation, one often uses a longer life-time medium to store the state of qubit. 2] Now quantum decoherence and quantum information-storage have been the central issues in the study of quantum information and computation.

Both long decoherence-time qubit and long life-time medium are potential candidates for quantum information. The traditional cavity QED system, 3] consisting of two-level atom and microwave cavity, can implement this quantum information-storage process. Now the decoherence time of Josephson junction qubit has been of order $10 \mu \mathrm{s}$. [4] Josephson junction qubit 5] can be coupled to superconducting microwave cavity [6] and transmission line resonator [7]. Recently, the integration of Josephson junction qubit and nanomechanical resonators [8] are attracting considerable attentions. These nanomechanical resonators can be easily fabricated using technologies of nanoelectronics (see, e.g., Refs [9, 10]). The dynamics of all these coupled systems could be described by the Jaynes-Cummings Hamiltonian. [11]

In contrast to nanomechanical resonator being assumed as a harmonic oscillator, the nonlinearity of nanomechanical resonator has attracted more and more attentions, 10] such as one can enhance the anharmonicity of nanomechanical resonators by subjecting them to inhomogenous electrostatic fields, [12] generating YurkeStoler states. [13] Here the coupled system including charge qubit and nonlinear nanomechanical resonator is considered.

*Electronic address: ybgao@bjut.edu.cn
This paper is organized as follows. In Sec. II, we describe the proposed model of a charge qubit interacting with a nonlinear nanomechanical resonator. In Sec. III, the nonlinearity of nanomechanical is considered in implementing the process of dynamical quantum information-storage. In Sec. VI, the influence of the nonlinearity of nanomechanical resonator on engineered decoherence is studied analytically.

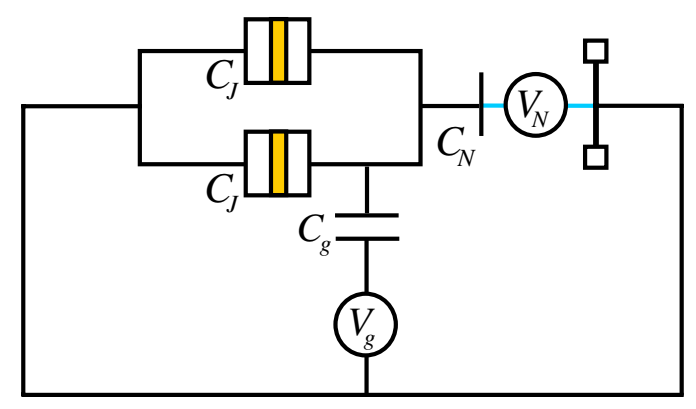

FIG. 1: Schematic diagram of a charge qubit capacitively coupled to a nanomechanical resonator. An externally applied voltage on the capacitor $\left(C_{N}\right)$ formed by the superconducting island of Josephson junction and the nanomechanical resonator. Here, $C_{J}$ and $C_{g}$ represent the capacitances for the Josephson junctions and the gate capacitor respectively. $V_{g}$ is the gate voltage applied to charge qubit via the gate capacitor, and $V_{N}$ is an externally applied voltage on the nanomechanical resonator.

\section{MODEL}

Due to the nonlinearity, the nanomechanical resonator is not assumed as harmonic resonator. The Hamiltonian for nonlinear nanomechanical resonator is described by 14

$$
H_{\mathrm{N}}=\frac{p^{2}}{2 m}+\frac{1}{2} m \omega^{2} x^{2}+\frac{1}{4} \alpha x^{4}
$$


where $m$ is the effective mass, $\omega$ is the linear resonator frequency and the parameter $\alpha$ measures the strength of nonlinearity. The canonical coordinate $x$ and momentum $p$ satisfies the commutation relation $[x, p]=i$. Hereafter, we assume $\hbar=1$. The $x$ and $p$ can be also represented by the annihilation and creation operators $\left(a\right.$ and $\left.a^{\dagger}\right)$,

$$
\begin{aligned}
& x=\frac{1}{\sqrt{2 m \omega}}\left(a^{\dagger}+a\right), \\
& p=i \sqrt{\frac{m \omega}{2}}\left(a^{\dagger}-a\right) .
\end{aligned}
$$

Thus the Hamiltonian in Eq. (10) can be rewritten as

$$
H_{N}=\omega a^{\dagger} a+\frac{\chi}{6}\left(a^{\dagger}+a\right)^{4}
$$

with the nonlinear parameter is $\chi=\alpha /(8 m \omega)$.

As shown in Fig. 1, with the oscillation of nanomechanical resonator, the Cooper pair box (charge qubit) is capacitively coupled to the nanomechanical resonator. Here $C_{N}(x)$ denotes the capacitance between nanomechanical resonator and the superconducting island of Cooper pair box. The distance $d$ between nanomechanical resonator and superconducting island is assumed much larger than the amplitude $x$ of the oscillation of nanomechanical resonator, i.e., $d \gg x$. In this case, one can approximately simplify the capacitance $C_{N}(x)$ as

$$
C_{N}(x) \simeq C_{N}\left(1-\frac{x}{d}\right) .
$$

According to the results in Refs. 15, 16], the Hamiltonian of charge qubit reads

$$
H_{c}=\frac{\omega_{q}}{2} \sigma_{z}+g\left(a+a^{\dagger}\right) \sigma_{x}
$$

with respect to the qubit frequency $\omega_{a}=E_{J}$ and the coupling constant $g=4 E_{C} n_{N} x_{z p} / d$. These spin operators $\left\{\sigma_{z}, \sigma_{ \pm}\right\}$are defined in the basis of charge qubit $\left\{|0\rangle_{q},|1\rangle_{q}\right\}$, i.e.,

$$
\sigma_{+}|0\rangle_{q}=|1\rangle_{q}, \quad \sigma_{-}|1\rangle_{q}=|0\rangle_{q} .
$$

Notice that the coupling constant $g$ is determined by the bias voltage $V_{N}\left(\propto n_{N}\right)$, and one can tune the qubit frequency $\omega_{q}$ through the external magnetic field. Then the total Hamiltonian for charge qubit and nonlinear nanomechanical resonator is

$$
H=H_{c}+H_{\mathrm{N}} .
$$

Where $H_{c}$ and $H_{\mathrm{N}}$ are given by Eqs. (416) respectively.

\section{REVIVAL OF QUANTUM COHERENCE}

During the process of the preparation, manipulation and measurement of quantum state, the loss and revival of quantum coherence is very important for building a real quantum computer. With a longer life-time medium (data bus), one can store, manipulate and communicate quantum information. [2] Considering the nonlinearity of nanomechanical resonator, the possibility of being quantum data bus in quantum computation will be explored in this section.

One can control the Josephson coupling energy $E_{J}$ such that the qubit is resonant with the nanomechanical resonator $\left(\omega_{q}=\omega\right)$. Using the rotating-wave approximation, the total Hamiltonian in Eq. (8) becomes

$$
H=\frac{\omega}{2} \sigma_{z}+g\left(a^{\dagger} \sigma_{-}+a \sigma_{+}\right)+(\omega+\chi) a^{\dagger} a+\chi\left(a^{\dagger} a\right)^{2} .
$$

It is just the same as the nonlinear Jaynes-Cummings Hamiltonian. 17] The nonlinear part in the above Hamiltonian $\chi\left(a^{\dagger} a\right)^{2}$ is obtained from the quartic potential $\left(\sim x^{4}\right.$ seen in Eq. (4) $)$. Here the nonlinear parameter $\chi$ is assumed as much smaller than the coupling constant $g$. When the nonlinear parameter $\chi$ vanishes, the Hamiltonian in Eq. (9) reduces into the Jaynes-Cummings Hamiltonian. [11] In the following, the influence of the nonlinear part $\chi\left(a^{\dagger} a\right)^{2}$ on quantum information storage and quantum coherence will be analyzed carefully.

Corresponding to the Hamiltonian in Eq. (9), we can solve the eigenvalues

$$
E_{ \pm}=\left(\frac{\omega}{2}+\chi\right) \pm g_{\chi}
$$

and the eigenstates

$$
\begin{aligned}
& |+\rangle=\cos \frac{\theta}{2}|01\rangle+\sin \frac{\theta}{2}|10\rangle, \\
& |-\rangle=-\sin \frac{\theta}{2}|01\rangle+\cos \frac{\theta}{2}|10\rangle .
\end{aligned}
$$

Where some parameters are defined as $g_{\chi}=\sqrt{g^{2}+\chi^{2}}$ and $\theta=\arcsin \left(g / g_{\chi}\right)$. Here the nonlinear parameter $\chi$ in Eq. (10) ) shows that the nonlinearity of nanomechanical resonator modifies the Rabi oscillation frequency $g_{\chi}$ in Jaynes-Cummings model.

Now the initial state of charge qubit is prepared in the coherent superposition state, $\alpha|0\rangle_{q}+\beta|1\rangle_{q}$, and the nanomechanical resonator in the vacuum state $|0\rangle_{N}$. Then the initial state of the total system writes

$$
|\psi(0)\rangle=\left(\alpha|0\rangle_{q}+\beta|1\rangle_{q}\right) \otimes|0\rangle_{N}
$$

for $|\alpha|^{2}+|\beta|^{2}=1$ and $|0\rangle_{q} \otimes|0\rangle_{N}=|00\rangle$. When the charge qubit begins to interact with the nanomechanical resonator, the state of the total system evolves into

$$
|\psi(t)\rangle=C_{00}(t)|00\rangle+C_{01}(t)|01\rangle+C_{10}(t)|10\rangle .
$$

Some parameters are given in the following

$$
\begin{aligned}
& C_{00}(t)=\alpha e^{-i \frac{\omega}{2} t}, \\
& C_{01}(t)=-i \beta \sin \theta e^{-i\left(\frac{\omega}{2}+\chi\right)} \sin \left(g_{\chi} t\right), \\
& C_{10}(t)=\beta e^{-i\left(\frac{\omega}{2}+\chi\right)}\left(\cos \left(g_{\chi} t\right)+i \cos \theta \sin \left(g_{\chi} t\right)\right)(.11)
\end{aligned}
$$


There exists the condition $\left|C_{00}\right|^{2}+\left|C_{01}\right|^{2}+\left|C_{10}\right|^{2}=1$.

The process of quantum information-storage can be implemented by

$$
\left(\alpha|0\rangle_{q}+\beta|1\rangle_{q}\right) \otimes|0\rangle_{N} \rightarrow|0\rangle_{q} \otimes\left(\alpha|0\rangle_{N}+\beta|1\rangle_{N}\right) .
$$

Considering the nonlinearity, the time evolution of the probability of quantum information transferred from qubit to nanomechanical resonator is

$$
\begin{aligned}
P & =\left|C_{00}(t)\right|^{2}+\left|C_{01}(t)\right|^{2} \\
& =|\alpha|^{2}+|\beta|^{2}\left[\sin \theta \sin \left(g_{\chi} t\right)\right]^{2} .
\end{aligned}
$$

When the nonlinearity is not considered in Eqs. (911) $(\chi=0)$, the probability $P$ is 1 . Then the quantum information can be transferred from charge qubit to nanomechanical resonator. Obviously seen in Eq. (11), the probability $P$ is smaller than 1 . Then the process of quantum information-storage can not be implemented and the nonlinear nanomechanical resonator can not be a quantum data bus.

At time $\tau=k \pi / g_{\chi}$ for $k \mathrm{~s}$ being integers, there exists $C_{01}(\tau)=0$ and the state of the total system evolves into

$$
|\psi(\tau)\rangle=\left(C_{00}(\tau)|0\rangle_{q}+C_{10}(\tau)|1\rangle_{q}\right) \otimes|0\rangle_{N}
$$

Here the quantum information ( $\alpha$ and $\beta$ ) returns into the qubit and the nonlinearity maintains the revival of quantum coherence of charge qubit.

\section{ENGINEERED DECOHERENCE}

In previous work, 3] the traditional cavity QED system (consisting of a two-level atom and a microwave cavity) demonstrates a reversible decoherence process of mesoscopic superposition of field states. This model (consisting of a charge qubit and a nonlinear nanomechanical resonator) will show the influence of the nonlinearity on its engineered decoherence.

According to Eq. (8), we turn off the Josephson coupling energy $E_{J}$, i.e., $\omega_{q}=0$. Thus the Hamiltonian corresponding to a standard quantum measurement model is obtained, [15]

$$
H=H_{0}|0\rangle\left\langle 0\left|+H_{1}\right| 1\right\rangle\langle 1| .
$$

where the effective Hamiltonian $H_{k}$ is

$$
H_{k}=(-1)^{k} g\left(a^{\dagger}+a\right)+\omega a^{\dagger} a+\frac{\chi}{6}\left(a+a^{\dagger}\right)^{4}
$$

with respect to the qubit state $|k\rangle$ (for $k=0,1$ ) which is the eigenvectors of spin operator $\sigma_{x}$. Obviously the Hamiltonian $H_{k}$ in Eq. (15) describes a driven nonlinear oscillator.

Assuming the initial state of the nanomechanical resonator in vacuum state and the qubit in a superposition state, then the initial state of the total system is

$$
|\psi(0)\rangle=(\alpha|0\rangle+\beta|1\rangle) \otimes|0\rangle_{N} .
$$

Here the time evolution of wave function for the total system is

$$
\begin{aligned}
|\psi(t)\rangle & =e^{-i H t}|\psi(0)\rangle \\
& =\alpha|0\rangle \otimes\left|\mu_{0}(t)\right\rangle+\beta|1\rangle \otimes\left|\mu_{1}(t)\right\rangle
\end{aligned}
$$

where we have defined

$$
\left|\mu_{k}(t)\right\rangle=e^{-i H_{k} t}|0\rangle_{N}
$$

For any coherent state, we have

$$
|\alpha\rangle=e^{\alpha a^{\dagger}-\alpha^{*} a}|0\rangle_{N}
$$

Applying the coherent displacement transformation

$$
D\left(\alpha_{k}\right)=e^{\alpha_{k} a^{\dagger}-\alpha_{k}^{*} a}
$$

and neglecting the fast oscillating terms, the Hamiltonian $H_{k}$ in Eq. (15) is transformed into the effective Hamiltonian

$$
H_{k}^{e f f}=D^{\dagger}\left(\alpha_{k}\right) H_{k} D\left(\alpha_{k}\right)=\Omega a^{\dagger} a+\chi\left(a^{\dagger} a\right)^{2}
$$

where the transformed angular frequency of nanomechanical resonator is $\Omega=\omega+\chi+8 \lambda^{2} \chi$ for $\alpha_{k}=(-1)^{k} \lambda=$ $(-1)^{k} g /(\omega+\chi)$. The state vector in Eq. (17) writes

$$
\left|\mu_{k}(t)\right\rangle=D\left(\alpha_{k}\right) e^{-i H_{k}^{e f f} t} D^{\dagger}\left(\alpha_{k}\right)|0\rangle_{N} .
$$

The process of quantum decoherence could be described by time evolution of the reduced density matrix of quantum system and the off-diagonal elements of reduced density matrix measures the decoherence. According to Eq. (16), the time evolution of density matrix for the total system is

$$
\rho(t)=|\psi(t)\rangle\langle\psi(t)|
$$

Then the reduced density matrix for charge qubit is calculated as

$$
\begin{aligned}
\rho_{s}(t)= & \operatorname{Tr}(|\psi(t)\rangle\langle\psi(t)|)) \\
= & \alpha^{*} \alpha|0\rangle\left\langle 0\left|+\left\langle\mu_{1}(t) \mid \mu_{0}(t)\right\rangle \alpha \beta^{*}\right| 0\right\rangle\langle 1| \\
& +\left\langle\mu_{0}(t) \mid \mu_{1}(t)\right\rangle \alpha^{*} \beta|1\rangle\left\langle 0\left|+\beta^{*} \beta\right| 1\right\rangle\langle 1| .(19)
\end{aligned}
$$

As a measure of decoherence, the off-diagonal elements of the reduced density matrix can be defined as decoherence factor [18]

$$
D(t)=\left|\left\langle\mu_{1}(t) \mid \mu_{0}(t)\right\rangle\right| .
$$

To clarify the influence of the nonlinearity of nanomechanical resonator, the decoherence factor will be calculated in the following.

Using the Baker-Hausdoff formulas, it is easily calculated that

$$
\begin{aligned}
U_{\chi}^{\dagger}(t) a U_{\chi}(t) & =e^{i \chi t a^{\dagger} a} a, \\
U_{\chi}^{\dagger}(t) a^{\dagger} U_{\chi}(t) & =a^{\dagger} e^{i \chi t a^{\dagger} a} .
\end{aligned}
$$


The decoherence factor becomes

$$
\begin{gathered}
D(t)=\mid\left\langle-\alpha_{1} e^{-i \Omega t}\right| U_{\chi}^{\dagger}(t) D^{\dagger}\left(\alpha_{1}\right) U_{\chi}(t) \\
U_{\chi}^{\dagger}(t) D\left(\alpha_{0}\right) U_{\chi}(t)\left|-\alpha_{0} e^{-i \Omega t}\right\rangle \mid
\end{gathered}
$$

where we have adopted $U_{\chi}(t) U_{\chi}^{\dagger}(t)=1$ for $U_{\chi}(t)=$ $e^{-i \chi\left(a^{\dagger} a\right)^{2} t}$.

Taking the coherent state displacement operator

$$
D(\xi)=e^{-\frac{1}{2}|\xi|^{2}} \sum_{k, m=0}^{\infty} \frac{\left(\xi a^{\dagger}\right)^{k}\left(-\xi^{*} a\right)^{m}}{k ! m !}
$$

and

$$
\left(e^{i \chi t a^{\dagger} a} a\right)^{m}|\xi\rangle=(\xi)^{m}\left(e^{i \chi t}\right)^{\frac{m(m-1)}{2}}\left|\xi e^{i m \chi t}\right\rangle
$$

into Eq. (20), the decoherence factor is obtained

$$
\begin{aligned}
D(t) & =\mid e^{-3 \lambda^{2}} \sum_{k, m=0}^{\infty} \frac{\left(2 \lambda^{2}\right)^{k+m}}{k ! m !}\left(e^{i \Omega t}\right)^{k}\left(e^{-i \chi t}\right)^{\frac{k(k-1)}{2}} \\
& \left(e^{-i \Omega t}\right)^{m}\left(e^{i \chi t}\right)^{\frac{m(m-1)}{2}} e^{-\lambda^{2}-\lambda^{2} e^{-i k \chi t} e^{i m \chi t}} \mid .
\end{aligned}
$$

Without the nonlinearity, $\chi=0$ and $\lambda=g / \omega$, the decoherence factor in Eq. (21) reduces into

$$
D(t)=e^{-8 \frac{g^{2}}{\omega^{2}} \sin ^{2}\left(\frac{1}{2} \omega t\right)} .
$$

Which is also obtained in the coupled system of charge qubit and microwave cavity. [19]

To demonstrate the influence of the nonlinearity on the quantum decoherence, we consider it in the shorttime limit. Here the condition $\chi t \ll 1$ is assumed, we get $e^{i \chi t} \approx 1+i \chi t$. Then the decoherence factor in Eq. (21) is approximately

$$
D(t)=e^{-8 \lambda^{2} \sin ^{2}\left[\frac{1}{2}\left(\Omega+\lambda^{2} \chi+\frac{1}{2} \chi\right) t\right]} .
$$

Through some simple calculations, we have $\Omega+\lambda^{2} \chi+$ $\frac{1}{2} \chi>\omega$ and $\lambda^{2}<g^{2} / \omega^{2}$. Seen in Eqs. (22, 23), the nonlinearity of nanomechanical resonator increases the speed of oscillation and reduces the amplitude of oscillation in $D(t)$.

\section{CONCLUSIONS}

Considering the nonlinearity of nanomechanical resonator, the possibility of implementing quantum information storage and quantum decoherence of charge qubit are studied analytically. Using nonlinear JaynesCummings model, we find that the nonlinear nanomechanical resonator can not be a quantum data bus. In addition, the equation (13) shows that the nonlinearity does not destroy the revival of quantum coherence of charge qubit. With the calculations of decoherence factor in Eqs. (21-23), we demonstrate the influence of the nonlinearity of nanomechanical resonator on engineered decoherence of charge qubit. It shows that the nonlinearity of nanomechanical resonator affects the oscillation in decoherence factor $D(t)$.

\section{Acknowledgments}

We thank Dr. M. Hua and Dr. X. Xiao for helpful discussions.
[1] J. A. Wheeler and Z. H. Zurek, Quantum Theory of Measurement, Princeton University Press, NJ (1983).

[2] D. DiVincenzo, Fortschr. Phys. 48 (2000) 771.

[3] J. M. Raimond, M. Brune, and S. Haroche, Rev. Mod. Phys. 73 (2001) 565.

[4] Hanhee Paik, D. I. Schuster, Lev S. Bishop, G. Kirchmair, G. Catelani, A. P. Sears, B. R. Johnson, M. J. Reagor, L. Frunzio, L. I. Glazman, S. M. Girvin, M. H. Devoret, and R. J. Schoelkopf, Phys. Rev. Lett. 107 (2011) 240501.

[5] Y. Makhlin, G. Schoen, and A. Shnirman, Rev. Mod. Phys. 73 (2001) 357.

[6] C. P. Yang, S. I. Chu, and S. Han, Phys. Rev. Lett. 92 (2004) 117902.

[7] A. Wallraff, D. I. Schuster, A. Blais, L. Frunzio, R. S. Huang, J. Majer, S. Kumar, S. M. Girvin, and R. J. Schoelkopf, Nature 431 (2004) 162.

[8] F. Xue, Y. D. Wang, C. P. Sun, H. Okamoto, H. Yamaguchi, and K. Semba, New J. Phy. 9 (2007) 35.

[9] M. D. LaHaye, J. Suh, P. M. Echternach, K. C. Schwab, and M. L. Roukes, Nature 459 (2009) 960.
[10] L. G. Villanueva, R. B. Karabalin, M. H. Matheny, D. Chi, J. E. Sader, and M. L. Roukes, Phys. Rev. B 87 (2013) 024304.

[11] E. T. Jaynes and F. W. Cummings, Proc. IEEE 51 (1963) 89.

[12] S. Rips, I. WilsonRae, and M. J. Hartmann, arXiv: 1206.0147.

[13] F. L. Semio, K. Furuya, and G. J. Milburn, Phys. Rev. A 79 (2009) 063811.

[14] S. M. Carr, W. E. Lawrence, and M. N. Wybourne, Phys. Rev. B 64 (2001) 220101.

[15] Y. D. Wang, Y. B. Gao, and C. P. Sun, Eur. Phys. J. B 40 (2004) 321.

[16] Y. B. Gao, S. Yang, Y. X. Liu, C. P. Sun, and F. Nori, arXiv:0902.2512

[17] P. Gora and C. Jedrzejek, Phys. Rev. A 45 (1992) 6816.

[18] C. P. Sun, Phys. Rev. A 48 (1993) 898. C. P. Sun et.al, Fortschr. Phys. 43 (1995) 585.

[19] Y. B. Gao and C. Li, Commun. Theor. Phys. 43 (2005) 213. 